\title{
Cotargeting survival signaling pathways in cancer
}

\section{Steven Grant}

\author{
Departments of Medicine and Biochemistry, Massey Cancer Center and the Institute for Molecular Medicine, \\ Virginia Commonwealth University Health Science Center, Richmond, Virginia, USA.
}

\begin{abstract}
Mammalian target of rapamycin (mTOR) is a component of a signaling pathway (PTEN/PI3K/AKT) that is frequently dysregulated in cancer. However, its precise relationship to the MAPK cascade (Ras/Raf/MEK/ERK), another pathway often implicated in tumorigenesis, has not been well defined. Recent evidence from tissue specimens obtained from patients who have received $m$ TOR inhibitors suggests that ERK may be activated in response to mTOR interruption. In this issue of the JCI, Waugh Kinkade et al. and Carracedo et al. examine the relationship between these pathways in prostate and breast cancer cell model systems (see the related articles beginning on pages 3051 and 3065, respectively). Their findings suggest a link between inhibition of $m$ TOR and ERK activation, possibly reflecting interruption of a novel negative S6K1-dependent feedback loop. Significantly, both groups observed that simultaneous inhibition of MEK/ERK and $m$ TOR resulted in substantially enhanced antitumor effects both in vitro and in vivo. Together, these findings suggest that concurrent interruption of complementary signaling pathways warrants further investigation in cancer therapy.
\end{abstract}

\section{Pathway addiction}

The dependence of transformed cells on the activation of survival signaling pathways has long been recognized. In the case of chronic myelogenous leukemia (CML), an apical lesion has been identified, namely, the BCR-ABL oncoprotein, a constitutively active tyrosine kinase that activates multiple other antiapoptotic pathways including NF- $\mathrm{KB}, \mathrm{AKT}$, and STAT5, among others (1). The discovery and identification of this mutant oncoprotein sparked the development of multiple BCR-ABL kinase inhibitors (e.g., imatinib mesylate, dasatinib, nilotinib), which have shown impressive activity in this disease. In addition, the success of BCR-ABL kinase inhibitors in CML raised the possibility that similar strategies might also be effective in other malignancies, including those of epithelial origin. However, efforts to translate the outstanding success of targeted agents in CML to the solid tumor field have been, with a few exceptions, problematic. In all likelihood, this reflects multiple interdependent factors. For example, in epithelial malignancies, while certain genetic abnormalities such as

Nonstandard abbreviations used: $\mathrm{CML}$, chronic myelogenous leukemia; mTOR, mammalian target of rapamycin; mTORC1, mTOR complex 1; PTEN, phosphatase and tensin homolog; S6K, S6 kinase.

Conflict of interest: The author has declared that no conflict of interest exists.

Citation for this article: J. Clin. Invest. 118:3003-3006 (2008). doi:10.1172/JCI36898. phosphatase and tensin homolog (PTEN) mutations may be very common (2), they do not appear to play the central role in tumor cell survival that mutant BCR-ABL appears to do in CML. In addition, even if a particular genetic abnormality is critical to the transformation process, other cooperating mutations may be required to allow such cells to survive. Recently, attention has focused on the concept of "oncogene addiction," a phenomenon in which mutant genes not only provide transformed cells with a survival advantage over their normal counterparts, but are also required to avoid cell death (3). A corollary of this hypothesis is that complementary survival pathways, once activated, may replace survival signals induced by the transforming oncogene, thereby relieving the cell of its addiction. The existence of such redundant survival pathways could also explain why inhibitors of a single dysregulated pathway may be insufficient to induce cell death in tumor cells. In support of this notion, recent studies demonstrated that in glioblastoma cells, inhibition of multiple, rather than a single, receptor tyrosine kinase(s) was necessary to induce cell death (4).

\section{Targeting the MAPK and mTOR pathways}

Two of the most important signaling cascades frequently dysregulated in cancer are the Ras/Raf/MEK/ERK (MAPK) and the PTEN/PI3K/AKT/mammalian target of rapamycin (mTOR) pathways (Figure 1). In addition, there is accumulating evidence that these pathways may cooperate to promote the survival of transformed cells (5). Activating mutations of Ras and Raf occur frequently in both solid tumor and hematologic malignancies, leading to activation of their downstream targets MEK1/2 and ERK1/2 (6). This has prompted the clinical development of small molecule inhibitors targeting specific components of the MAPK cascade, such as farnesyltransferase inhibitors (e.g., tipifarnib), Raf- 1 inhibitors (e.g., sorafenib), and MEK1/2 inhibitors (e.g., AZD6244). Similarly, the PI3K/AKT/ mTOR pathway is one of the most frequently mutated pathways in solid tumor malignancies. For example, mutation/inactivation of PTEN often occurs in such tumors, resulting in activation of PI3K and its downstream targets AKT and mTOR (7). While the development of PI3K and AKT inhibitors is currently the focus of intense interest, the bulk of clinical experience has been obtained with inhibitors of mTOR such as rapamycin and its derivatives. mTOR integrates diverse signals mediated by growth factors, energy requirements, and cellular stress to regulate protein translation and survival. It acts through its association with two distinct signaling complexes: MTOR complex $1 /$ regulatory-associated protein of mTOR (mTORC1/RAPTOR), which responds to growth factors and nutrient availability and is inhibited by rapamycin, and mTORC2/rapamycin-insensitive companion of mTOR (RICTOR), which primarily responds to growth factors and is rapamycin insensitive (8). Interestingly, a negative feedback loop has been described in which inhibition of mTORC1 by rapamycin results in AKT activation (9), although in other systems, prolonged rapamycin exposure may inhibit TORC 2 assembly and disrupt AKT signaling (10). In contrast, effects of mTOR inhibition on the MAPK pathway have not been well defined.

\section{MAPK and mTOR interactions}

Evidence of crosstalk between ERK and mTOR (11) raised the possibilities that a feedback loop might exist in this network, 


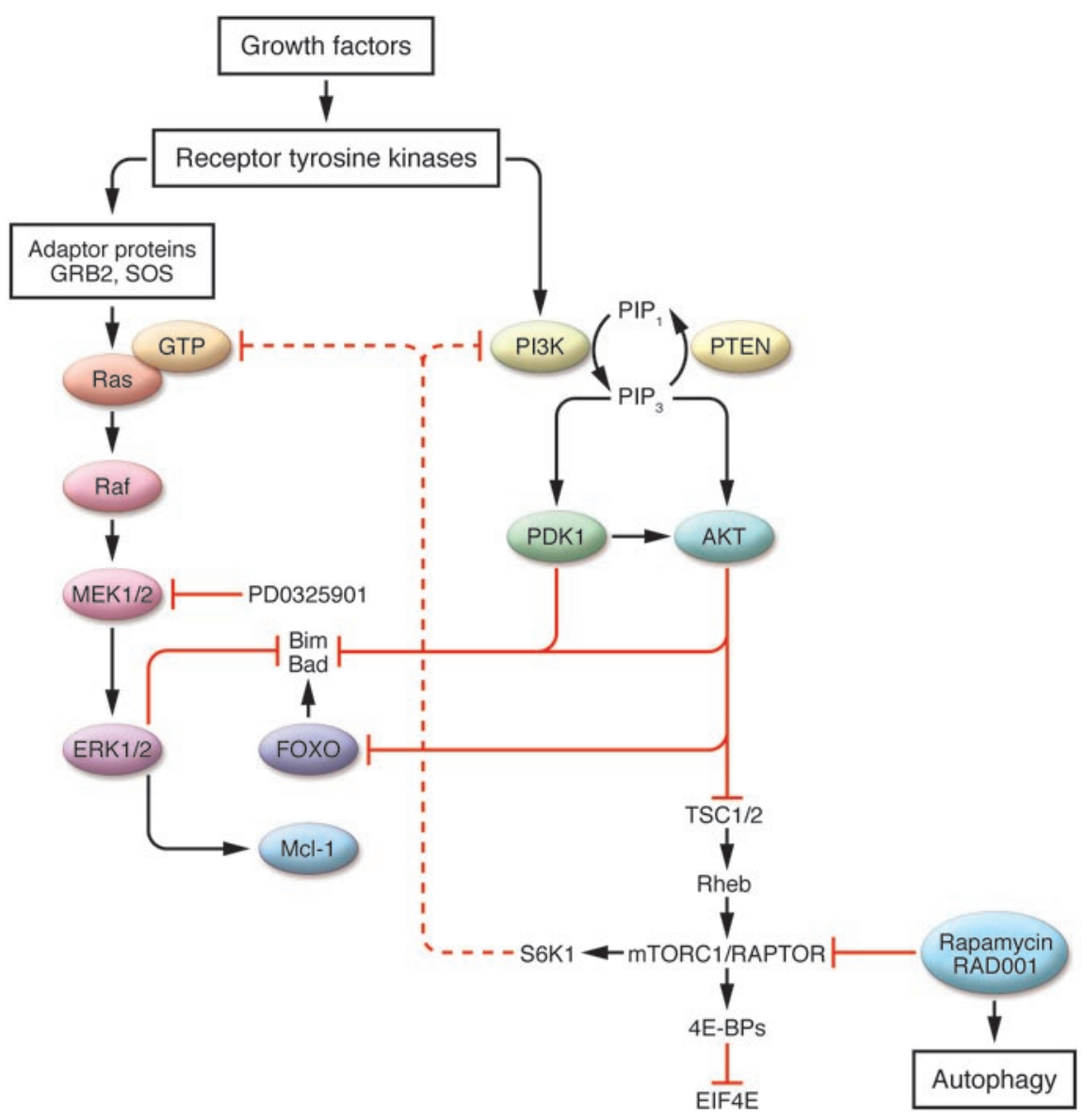

Figure 1

Diagrammatic representation of the MAPK and the PTEN/PI3K/AKT/mTOR pathways and their potential interactions in transformed cells. Growth factor and other survival signals are transduced through receptor tyrosine kinases via adaptor proteins (such as GRB2 and the guanine-nucleotide exchange factor SOS) and activate Ras and its downstream targets, Raf and MEK1/2, which results in the activation of ERK1/2. ERK1/2 activation leads to perturbations in downstream targets that promote survival, i.e., downregulation of Bim and Bad and upregulation of myeloid cell leukemia sequence 1 (Mcl-1). Growth factor survival signaling proceeds through the PI3K pathway, a process that is amplified in cells deficient in the PTEN phosphatase. PI3K activates pyruvate dehydrogenase kinase isozyme 1 (PDK1) and AKT, which either directly or indirectly (i.e, via forkhead box O [FOXO]) inactivate proapoptotic molecules such as Bim and Bad. AKT also activates mTORC1/regulatory-associated protein of mTOR (RAPTOR) by relieving it of the inhibitory influence of the tuberous sclerosis $1 / 2$ (TSC1/2) complex. It is postulated that pharmacologic inhibition of mTOR (e.g., by rapamycin or RAD001) leads, via an S6K1- and PI3K-dependent process, to activation of Ras and MEK/ERK, which promotes cell survival in the face of mTOR inhibition. Inhibition of mTOR may also provoke an autophagic response. Conversely, interruption of the MAPK pathway (i.e., by MEK1/2 inhibitors such as PD0325901) in the setting of mTOR inhibition promotes cell death through a Bim-dependent mechanism. 4EBP, EIF4E-binding protein; EIF4E, eukaryotic translation initiation factor 4E; GRB2, growth factor receptor-bound protein 2; PIP ${ }_{1}$, phosphatidylinositol 3-phosphate; PIP $_{3}$, phosphatidylinositol-3,4,5-triphosphate.

and that if this is the case, such a phenomenon might be exploited therapeutically. The results of two separate studies in the current issue of the JCI support these notions. In the report by Waugh Kinkade et al. (12), the authors employed a PTENdeficient transgenic prostate cancer model to investigate the effects of simultaneous ally related to upregulation of Bim, a proapoptotic BH3-only member of the Bcl-2 family whose abundance is known to be upregulated by MEK/ERK inactivation (13). In patient specimens, activation of ERK and the PTEN/PI3K/AKT/mTOR pathway was associated with prostate cancer progression; moreover, the authors found that combined MEK/ERK and mTOR inhibition was effective in the adjuvant setting. An interesting and somewhat unexpected finding was that the inhibitory effects of this regimen on prostate cancer cell growth were primarily directed against androgen-independent, rather than-dependent, disease. The authors concluded that a strategy combining MEK/ERK and mTOR inhibition may be effective in the treatment of advanced cancer or in the adjuvant setting, particularly in the case of androgenindependent disease.

In a parallel study, Carracedo et al. (14) reported that tumor cell biopsies obtained from patients treated in the neoadjuvant setting with the mTOR inhibitor RAD001 displayed activation of ERK. Similar events were observed in a breast cancer cell model. The involvement of a negative regulatory S6 kinase (S6K)/PI3K/AKT/mTOR-dependent pathway in ERK activation was confirmed by both pharmacologic and genetic means. Interestingly, the inhibitory effects of this strategy on prostate cancer cell growth in the in vitro setting were not clearly related to apoptosis induction, whereas in animal studies, a clear increase in apoptosis was observed. The authors concluded that exposure of cancer cells to mTORC1 inhibitors results in a compensatory activation of the MAPK pathway via release of an S6K1-related "brake" on PI3K and Ras and that interruption of ERK activation by pharmacologic agents significantly increases the efficacy of clinically relevant mTOR inhibitors. A summary of these interactions is depicted in Figure 1.

\section{Questions and future perspectives}

These provocative findings have obvious translational implications for the treatment of prostate and breast cancer and potentially other malignancies. However, both studies raise a number of questions that will require considerable work to answer. In each study, a key unresolved issue is the precise mechanism by which simultaneous interruption of the MAPK and mTOR pathways exerts its antitumor effects and promotes cell death. In this context, it is tempting to relate perturbations in signal- 
ing pathways to their effects on members of the Bcl-2 family of pro- and antiapoptotic proteins. For example, She et al. reported that in breast cancer cells, EGFR/MAPK inhibitors cooperated with PI3K inhibition to induce cell death by dephosphorylating the proapoptotic protein Bad at two separate serine phosphorylation sites; concurrent disruption of these phosphorylations spare the protein from proteasomal degradation (15). Thus, in this case, death signals mediated by simultaneous interruption of the MAPK and PTEN/PI3K/AKT/mTOR pathways are integrated through $\mathrm{Bad}$. In view of the known regulation of Bim by both the MAPK (13) and the AKT pathways (16), it is also logical to postulate that Bimrelated mechanisms might be involved in the regulation of cancer cell survival and proliferation in the face of MEK/ERK and mTOR inhibitors. In support of this notion, Waugh Kinkade et al. found that Bim expression was markedly increased in cells exposed to both agents and that this phenomenon played a key role in the antitumor effects of the regimen (12). One puzzling finding in the study by Carracedo is that there was no clear increase in apoptosis in cells simultaneously exposed to inhibitors in vitro, whereas apoptosis was pronounced in tumor cells exposed to the agents in vivo (14). A possible explanation for this phenomenon is that inhibition of mTOR is known to promote autophagy, a process that under some circumstances antagonizes cell death (17). If this process is operative primarily in the in vitro setting, it could potentially explain the discordance between apoptosis induction in vitro and in vivo observed by Carracedo et al. An alternative possibility is that concurrent MAPK and mTOR inhibition may cooperate to disrupt tumor angiogenesis, which could promote cancer cell death in vivo through an indirect mechanism. Clearly, additional studies will be required to resolve these questions.

Another interesting and potentially significant finding was that the strategy of simultaneously interrupting MAPK and mTOR signaling was primarily active against androgen-independent prostate cancer cells, which are characteristically more resistant to therapy than their androgen-dependent counterparts. Although much is known about survival signaling pathways in androgen-dependent prostate cancer cells (18), much less is known about signaling networks in cells that survive and proliferate independently of androgen stimulation. However, recent studies indicate that activation of the MEK/ERK and $\mathrm{PTEN} / \mathrm{PI} 3 \mathrm{~K} / \mathrm{AKT} / \mathrm{mTOR}$ pathways may play a key role in the survival of such androgen-independent prostate cancer cells (19). In view of the relative paucity of treatment options for patients with androgen-independent disease, a strategy specifically targeting pathways essential for the survival of such cells is particularly attractive.

Other key issues remaining to be resolved include the identification of the mechanism(s) responsible for the feedback loop in and crosstalk between the MAPK and PTEN/PI3K/AKT/mTOR pathways and the selection of specific inhibitory strategies to achieve optimal therapeutic benefits. As noted previously (4), activation of multiple parallel survival cascades may require simultaneous interruption of more than one signaling pathway to induce tumor cell death. It is tempting to speculate that in tumor cell development, cells that exhibit activation of multiple survival pathways enjoy a survival benefit over those that do not and are eventually able to predominate due to selection pressure. However, this may represent a phenomenon fundamentally different from that described by Carracedo et al. (14), in which a disruption of an S6K1-related negative feedback loop, leading to PI3K activation, was postulated to account for Ras-related ERK activation in the face of TORC1 inhibition. Under these conditions, activation of ERK may represent a cytoprotective response to interruption of what might otherwise be lethal interruption of mTOR signaling. It remains to be determined whether inhibition of a constitutively active versus a stimulated complementary pathway will prove to be the more effective strategy.

Finally, a question arises concerning the optimal strategy to inhibit multicomponent pathways. Currently, mTOR inhibitors such as rapamycin and its derivatives have been the agents of choice for interruption of the PTEN/PI3K/AKT/mTOR pathway in view of their clinical availability, but this may change with the entry of PI3K inhibitors into the clinic and ongoing development of AKT inhibitors. The choice is one of theoretical as well as practical significance. For example, as noted above, the effects of mTOR inhibition on AKT activation status may vary with cell type and context and may lead to either up- or downregulation of this pathway (9, 10). In addition, PI3K has downstream targets other than AKT, and AKT signals to numerous proteins in addition to $\mathrm{mTOR}$ (20). It will be important to determine whether and under what circumstances regimens combining MEK/ERK inhibitors with inhibitors of PI3K, AKT, or mTOR will prove to be the optimal strategy. Similar considerations may also apply to inhibitors of targets upstream of MEK/ ERK (e.g., Raf-1, farnesyltransferase, etc.). Whatever the answers to these questions, it is likely that in the future, aside from efforts to improve the activity of conventional cytotoxic drugs through combination strategies involving targeted agents, significant attention will also be directed toward the rational combination of novel agents that disrupt complementary tumor cell survival pathways. In all likelihood, these initiatives will have important implications for cancer therapy.

\section{Acknowledgments}

The author is supported by awards CA63753, CA93798, and CA100866 from the NIH, the Multiple Myeloma Research Foundation, and the V Foundation.

Address correspondence to: Steven Grant, Room 234 Goodwin Research Building, 401 College Street, Richmond, Virginia 23298, USA. Phone: (804) 828-5211; Fax: (804) 828-2174; E-mail: stgrant@vcu.edu.

1. Van Etten, R.A. 2007. Oncogenic signaling: new insights and controversies from chronic myeloid leukemia. J. Exp. Med. 204:461-465.

2. Cantley, L.C., and Neel, B.G. 1999. New insights into tumor suppression: PTEN suppresses tumor formation by restraining the phosphoinositide 3-kinase/AKT pathway. Proc. Natl. Acad. Sci. U. S. A. 96:4240-4245.

3. Weinstein, I.B., and Joe, A.K. 2006. Mechanisms of disease: oncogene addiction - a rationale for molecular targeting in cancer therapy. Nat. Clin. Pract. Oncol. 3:448-457.

4. Stommel, J.M., et al. 2007. Coactivation of receptor tyrosine kinases affects the response of tumor cells to targeted therapies. Science. 318:287-290.

5. McCubrey, J.A., et al. 2006. Roles of the RAF/MEK/ ERK and PI3K/PTEN/AKT pathways in malignant transformation and drug resistance. Adv. Enzyme Regul. 46:249-279.

6. Sebolt-Leopold, J.S., and Herrera, R. 2004. Targeting the mitogen-activated protein kinase cascade to treat cancer. Nat. Rev. Cancer. 4:937-947.

7. Manning, B.D., and Cantley, L.C. 2007. AKT/ $\mathrm{PKB}$ signaling: navigating downstream. Cell. 129:1261-1274.

8. Bjornsti, M.A., and Houghton, P.J. 2004. The TOR pathway: a target for cancer therapy. Nat. Rev. Cancer. 4:335-348.

9. O'Reilly, K.E., et al. 2006. mTOR inhibition induces upstream receptor tyrosine kinase signaling and activates Akt. Cancer Res. 66:1500-1508.

10. Sarbassov, D.D., et al. 2006. Prolonged rapamycin treatment inhibits mTORC2 assembly and Akt/ PKB. Mol. Cell. 22:159-168.

11. Mitsiades, N., et al. 2002. Biologic sequelae of nucle- 
ar factor-kappaB blockade in multiple myeloma: therapeutic applications. Blood. 99:4079-4086.

12. Kinkade, C.W., et al. 2008. Targeting AKT/mTOR and ERK MAPK signaling inhibits hormone-refractory prostate cancer in a preclinical mouse model. J. Clin. Invest. 118:3051-3064.

13. Ley, R., et al. 2004. Extracellular signal-regulated kinases $1 / 2$ are serum-stimulated "Bim(EL) kinases" that bind to the $\mathrm{BH} 3$-only protein $\mathrm{Bim}(\mathrm{EL})$ causing its phosphorylation and turnover. J. Biol. Chem. 279:8837-8847.

14. Carracedo, A., et al. 2008. Inhibition of mTORC1 leads to MAPK pathway activation through a PI3Kdependent feedback loop in human cancer. J. Clin. Invest. 118:3065-3074.

15. She, Q.B., et al. 2005. The BAD protein integrates survival signaling by EGFR/MAPK and PI3K/Akt kinase pathways in PTEN-deficient tumor cells. Cancer Cell. 8:287-297.

16. Qi, X.J., Wildey, G.M., and Howe, P.H. 2006. Evidence that Ser87 of BimEL is phosphorylated by Akt and regulates BimEL apoptotic function. J. Biol. Chem. 281:813-823.

17. Mathew, R., Karantza-Wadsworth, V., and White, E.
2007. Role of autophagy in cancer. Nat. Rev. Cancer. 7:961-967.

18. Kaarbo, M., Klokk, T.I., and Saatcioglu, F. 2007. Androgen signaling and its interactions with other signaling pathways in prostate cancer. Bioessays. 29:1227-1238.

19. Shen, M.M., and Abate-Shen, C. 2007. Pten inactivation and the emergence of androgen-independent prostate cancer. Cancer Res. 67:6535-6538.

20. Datta, S.R., Brunet, A., and Greenberg, M.E. 1999. Cellular survival: a play in three Akts. Genes Dev. 13:2905-2927.

\title{
The elusive physiologic role of Factor XII
}

\author{
Alvin H. Schmaier \\ Division of Hematology and Oncology, Case Western Reserve University and University Hospitals Case Medical Center, Cleveland, Ohio, USA.
}

\begin{abstract}
Physiologic hemostasis upon injury involves many plasma proteins in a well-regulated cascade of proteolytic reactions to form a clot. Deficiency of blood coagulation Factors VIII, IX, or XI is associated with hemophilia. Factor XII (FXII) autoactivates by contact with a variety of artificial or biologic negatively charged surfaces (contact activation), resulting in blood coagulation and activation of the inflammatory kallikrein-kinin and complement systems. However, surprisingly, individuals deficient in FXII rarely suffer from bleeding disorders. Most biologic surfaces that activate FXII become expressed in disease states. Investigators have long searched for physiologic activators of FXII and its role in vivo. In this issue of the JCI, Maas et al. show that misfolded protein aggregates produced during systemic amyloidosis allow for plasma FXIIa and prekallikrein activation and increased formation of kallikrein-C1 inhibitor complexes, without Factor XIa activation and coagulation (see the related article beginning on page 3208). This study describes a novel biologic surface for FXII activation and activity, which initiates inflammatory events independent of hemostasis.
\end{abstract}

In the early 1950s, Oscar Ratnoff and Joan Colopy observed a patient, John Hageman, whose blood, upon routine preoperative screening, was found to have prolonged clotting times in glass test tubes, even though Hageman had no history or symptoms of a bleeding disorder (1). The observation that something was missing in his blood, and that this factor changed upon exposure to glass, ushered in the notion that blood clotting factors circulate as inactive precursors that can be activated. Ratnoff, in collaboration with Earl Davie, identified that, in the disorder that became known as Hageman trait, a plasma serine protease later called Factor XII (FXII) was missing. Absence of FXII prevents the activation of the blood coagulation zymogen

Nonstandard abbreviations used: FXII, Factor XII; FXIIa, activated FXII; HK, high-molecular-weight kininogen; PK, prekallikrein.

Conflict of interest: The author has declared that no conflict of interest exists.

Citation for this article: J. Clin. Invest. 118:3006-3009 (2008). doi:10.1172/JCI36617.
FXI that, when activated to become FXIa, leads to the formation of Factor IXa $-\mathrm{a}$ key intermediary in the intrinsic pathway of coagulation. These seminal studies contributed to the presentation of their waterfall cascade hypothesis for the blood coagulation system; a similar hypothesis was proposed that same year by Robert MacFarlane $(2,3)$. Ratnoff and his collaborators went on to show that FXII, which alters its physical properties during activation, induces vasodilatation and vascular permeability. These studies encapsulate the major known properties of FXII (Figure 1), a protein that autoactivates upon exposure to negatively charged surfaces to become the enzyme Factor XIIa ( $\alpha$-FXIIa), which then activates FXI, prekallikrein (PK), and C1 esterase (a subunit of the complement cascade). The consequence of FXI activation by $\alpha$-FXIIa is the initiation of a series of proteolytic reactions resulting in thrombin generation, which precedes clot formation. $\alpha$-FXIIa activation of PK forms plasma kallikrein that can reciprocally activate more FXII and liberate bradykinin from high-molecularweight kininogen (HK). Bradykinin is a mediator of vasodilatation and increased vascular permeability (4). $\alpha$-FXIIa when cleaved by plasma kallikrein forms Factor $\beta$ XIIa ( $\beta$-FXIIa), which then activates the macromolecular complex of the first component of complement, resulting in classic complement system activation; plasma kallikrein also directly activates complement components C 3 and C5 $(5,6)$. Thus, the activation of FXII results in coagulation and complement activation with bradykinin liberation (Figure 1).

\section{Contact activation of Factor XII}

Since FXII-deficient patients, along with PK- and HK-deficient patients, do not have a bleeding disorder, the relevance of the waterfall cascade hypothesis for blood coagulation hemostasis initiated by activated FXII has been questioned. Contact activation describes the unique property of FXII to undergo autoactivation and a change in shape when exposed to negatively charged artificial or biologic surfaces. Formation of FXIIa leads to PK activation in the presence of HK, hence the name contact activation system. However, the molecular basis for the formation of activated FXII remains unknown $(7,8)$. Over the last 4 decades, a growing list of "physiologic" negatively charged surfaces upon which FXII autoactivates have been recognized. In addition to nonphysiologic agents such as glass, polyethylene, and silicone rubber, FXII autoactivation occurs upon exposure to articular cartilage, skin, fatty acids, endotoxin, amyloid protein, and heparins, among others (9). It is in this context of identifying a biologic surface that sup- 